\title{
3D Morphology of Internal Defects in Wooden Products Based on Computed Tomography
}

\author{
Guiling Zhao, ${ }^{\mathrm{a}}$ Chang Liu, ${ }^{\mathrm{a}, *}$ Zhaowen Qiu, ,,c, Zongji Deng, ${ }^{\mathrm{b}}$ and Jinhua Gong ${ }^{\mathrm{d}}$ \\ A visualization method was used in this work for the 3D morphology of \\ internal defects in wooden products using a Philips Brilliance 16 computed \\ tomography $(\mathrm{CT})$. To obtain a high-quality $3 \mathrm{D}$ digital model, the original \\ images from the CT scan were segmented to manually color the cross- \\ and vertical sections of the wooden specimen. Through coloring, the \\ reconstructed model showed the accurate 3D morphology of internal \\ defects, such as cracks, wormholes, and decay parts, as well as clear \\ shapes of borer excrement, nails, bark, and wood parts of the specimen. \\ The results suggest that this method provides precise 3D models of \\ different types of defects in the wooden specimen. It can also accurately \\ measure the size and angle of the defects at any position for further \\ observation. This method can be effectively used for non-destructive \\ testing of wooden products and wooden cultural relics and can provide \\ accurate scales of defects and intuitive 3D models for wooden products \\ and wooden cultural relics restoration.
}

Keywords: Three-dimensional cultural wooden relics segmentation; Computed tomography; Total variation; $3 D$ reconstruction

Contact information: a: Northeast Agricultural University, Harbin, P.R. China, 150040; $b$ : Northeast Forestry University Harbin, China, 150040; c: HeiLongjiang Tuomeng Technology Co., Ltd.; d: Department of Biomedical Sciences, City University of Hong Kong; *Corresponding author: 1368817540@qq.com

\section{INTRODUCTION}

There are a large number of wooden cultural relics in the world, mainly including wooden buildings and furniture. These wooden cultural relics may be discolored, cracked, decayed by natural damage, such as wind, sun, rain, insects, etc., plus incur man-made damage, and their quantity decreases daily. Wooden cultural relics, such as ancient buildings and furniture, are often in good appearance, but the interior may have been damaged. Accurately judging the location, 3D shape, and precise scale of internal damage in wooden cultural relics is helpful for interventional protection. Therefore, the study of the 3D morphology of internal defects in wooden products is important.

At present, the method of research on internal defects of wood and wooden products mainly focuses on non-destructive testing using stress wave technology, and such methods are affected by wood temperature, moisture content, and compressive strength, and the accuracy is not high enough ( $\mathrm{Li}$ 2016; Gao et al. 2018). In 2010, Marino et al. used acoustic tomography to detect the pith location of chestnut. They analyzed the velocity gradient by correlating the longitudinal stress wave velocity value with the distance from the velocity measurement point to the pulp. It was demonstrated that the longitudinal velocity had a statistically significant relationship with the distance from the center of a log. In 2010, Hasegawa et al. (2010) applied the acoustoelastic technique to the nondestructive stress analysis of wood structures and carried out an experimental study on the acoustoelastic 
effect of Japanese fast-growing trees. Silva et al. (2014) used a nondestructive technique, with application of stress waves, for the evaluation of hardness of timber species in the Central Amazon. Liu et al. (2015) proposed a mathematical model of stress wave propagation on the cross-section of healthy trees and in 2017, Zhang et al. established a model of the propagation velocity of stress waves on the longitudinal section of wood, which has a certain contribution for the development of wood internal imaging technology. Li et al. (2016) stated that the stress wave velocity patterns can be used to diagnosis internal defects in urban trees and improve the accuracy of 3D tomographic images in tree inspection applications. Chen et al. (2016) evaluated the mechanical properties of wood by nondestructive testing. Non-destructive and static mechanical tests of Cunninghamia lanceolata and Ulmus rubra were carried out. With the development of computer technology in recent years, researchers have tried to use algorithms to obtain internal images of wooden specimen via stress wave detection (Du 2015; Wei et al. 2019). In 2015, Chen et al. proposed a 3D stress wave imaging method for wood internal defects based on the Top-k Inverse Distance Weighted (TIDW) algorithm, and initially obtained the 3D images of the defects. Du et al. (2019) established a 3D stress wave imaging method based on the Kriging algorithm. The average accuracy reached $89.1 \%$, which can roughly reflect the location of internal defects and the degree of damage in wood. Referred to the building formula, Chang et al. (2021) made 131 small specimens and 10 scaled timber column specimens to study the prediction of compressive bearing capacity of internal defects in ancient wooden buildings. There have been some studies on the relationship between the small specimens' wave resistance modulus and mechanics, non-destructive testing, and internal defects wood column compressive bearing capacity prediction, as well as a dangerous section judgment, and so on.

Computer tomography (CT) technology is mainly used in the medical field, and few studies have reported its application in wood and wooden cultural relic internal defects detection. For example, Ekevad (2004) used a series of pixel information of 2D CT images to calculate and analyze the internal fiber condition of the wood. Tsarouchas and Markaki (2011) proposes a method for extracting reliable architectural characteristics from complex porous structures using micro-computed tomography ( $\mu \mathrm{CT}$ ) images. Brodersen (2013) reviewed the traditional methods to reconstruct the origin of xylem, the application status of micro-computed tomography (mu-CT) in plant biology, and its technical issues. The mu-CT imaging method provides a new perspective for wood anatomy and emphasizes the importance of the relationship between wood structure and function. The authors' previous study (Zhao et al. 2015) performed a 3D reconstruction of Guqin cavity and converted 2D CT images into 3D models (The instrument used in the test is General Electric (GE) Brightspeedexcel4 spiral CT). This method can display the complete internal structure of the Guqin and obtain the size information of the Guqin cavity, which can provide technical support for the production process and quality inspection, but its internal visualization direction is limited. A later study (Zhao et al. 2018) used CT technology (Philips 16 slice spiral CT produced in Holland was used in the experiment) to obtain a high-precision 3D model of wooden cultural relics. This method can perform virtual segmentation at any position and any angle inside the wooden cultural relics. After segmentation, the internal wood structure can be clearly visualized, and the parameter value can be adjusted to distinguish the material density, but the 3D defect morphology inside the wood specimen was not studied. The purpose of this study is to investigate the 3D morphology of internal defects in wooden products using CT technology. 


\section{Experimental Preparation}

Wooden specimens were tested using a Philips Brilliance 16 CT (model A; Amsterdam, Netherlands). The parameters of the CT are listed in Table 1. The CT images of the defective parts were segregated by Matlab (MathWorks, v.2016, Natick, MA, USA). In the diagnostic image processing software, the cross-section images were generated by analyzing the vertical section images. Each CT image contains the corresponding sample coordinate information. After importing the DICOM3.0 format of CT image into the diagnostic image processing software, the coronal and sagittal images can be obtained. It can be understood as a book, in which each page is a data set. One can imagine the pages of the book compiled together. As in the case of the book, information can be located either front to back or relative to the sides. The artificial colouring process was completed by use of software from Heilongjiang Tuomeng Technology Co., Ltd., Harbin, China. All types of detects of the specimens were visualized using the $3 \mathrm{D}$ visual film reading terminal (Heilongjiang Tuomeng Technology Co., Ltd., Harbin, China).

Table 1. Philips Brilliance 16 CT (model A) Main Parameters

\begin{tabular}{|c|c|}
\hline Parameters & Value \\
\hline Fastest scanning speed & $360^{\circ}: 0.4 \mathrm{~s}$ \\
\hline Number of layers per scan & 16 \\
\hline Minimum layer thickness & $0.80 \mathrm{~mm}$ \\
\hline Minimum image reconstruction layer thickness & $0.6 \mathrm{mmu}$ \\
\hline Maximum layer thickness & $12 \mathrm{~mm}$ \\
\hline Image reconstruction speed & $6 \mathrm{images} / \mathrm{s}$ \\
\hline Scanning area & $25 \mathrm{~cm} \times 50 \mathrm{~cm}$ \\
\hline Image reconstruction matrix & $1024 \times 1024$ (optional) \\
\hline Maximum scanning time per single spiral & $100 \mathrm{~s}$ \\
\hline Maximum range per single spiral & $150 \mathrm{~cm}$ \\
\hline Orientation & All directions \\
\hline Locating image length & $175 \mathrm{~cm}$ \\
\hline
\end{tabular}

Four steps were prepared for the experiment. The first step was to obtain CT scanning images using the settings in Table 1. The CT scan test was completed in the CT scan room of the tertiary hospital (Harbin, China) with the same environmental conditions of a patient CT scan test. The second step used Matlab (MathWorks, v.2016, Natick, MA, USA) for image segmentation experiments in a Windows 7 operating system (Microsoft Corp., Redmond, WA, USA) to verify the effectiveness of this method. In the third step, the candidate cross- and vertical sections of CT images were manually colored in the selfdeveloped software (Diagnostic image processing software, Heilongjiang Tuomeng Technology Co., Ltd., Harbin, China) for further 3D defect morphology analysis. The fourth step was to complete the 3D reconstruction of the defect morphology in the specimen from multiple perspectives by adjusting settings, such as transparency and display status, in the patented software independently developed by Tuomeng Technology (3D visual film reading terminal, Heilongjiang Tuomeng Technology Co., Ltd., Harbin, China).

\section{Materials}

Two wooden specimens were tested in this study, and the basic information is shown in Table 2. Sample 1 was obtained from unused materials from the Wood Science Laboratory of Northeast Forestry University (Harbin, China). Sample 2 was purchased 
from Hongcun tourist souvenir store of Yi County (Huangshan City, Anhui Province, China).

Table 2. Basic Information of Specimens

\begin{tabular}{|c|c|c|c|c|}
\hline Specimen & $\begin{array}{c}\text { Size (Length } \times \text { Width } \times \\
\text { Height) }\end{array}$ & Material & Finishing & Carving \\
\hline $\mathrm{N} 1$ & $\begin{array}{c}\text { Height } 40.08 \mathrm{~cm} \\
\text { Volume } 9749 \mathrm{~mL}\end{array}$ & Log with bark & No paint & No carving \\
\hline $\mathrm{N} 2$ & $46 \mathrm{~cm} \times 22 \mathrm{~cm} \times 13 \mathrm{~cm}$ & Dalbergia & No paint & $\begin{array}{c}\text { Openwork, anaglyph, } \\
\text { diaglyph }\end{array}$ \\
\hline
\end{tabular}

Note: N1 has irregular width and length; N2 is a Noble Consort couch model that has 2 nails, 2 pieces of crack, multiple wormholes, and decayed parts

\section{D Visualization}

The defects in the specimen have similar CT image grayscales, and some of them connect to each other, which raises the difficulty to distinguish them. It is reported that TVL1 algorithm has good edge protection and multi-scale decomposition property that can solve the grayscale and connection problem; thus it was used to conduct the 3D visualization experiment. In order to have higher accuracy, the TVL1 was improved to a 3D-TVL1 version based on the edge-weighted total variation model,

$$
T V_{g}(u)=\grave{\mathrm{O}}_{\mathrm{w}} g|\tilde{\mathrm{N}} u| d x d y
$$

as proposed by Brodersen (2013) et al. The 3D-TVL1 algorithm inherits the property of TVL1 algorithm and has fast calculation speed and high locating accuracy. It is widely used in the field of medical image segmentation, and it is also suitable for wooden product CT image segmentation (Zhao et al. 2017). With the introduction of the discrete method and fast primal-dual projection, the 3D-TVL1 segmentation model can provide an ideal CT image segmentation effect.

There are mainly three steps to complete the 3D visualization process. Firstly, the 3D-TVL1 is used to segment the CT images of a wooden specimen. Then, the defect parts of the candidate CT images are labelled after the segmentation process. In this step, the decay xylem, wormholes, and cracks are considered as the internal morphology objectives and the bark, borer excrement, and iron nails are considered as the component morphology objectives. Finally, the labeled images are used to conduct 3D reconstruction. This process is summarized in the flow chart in Fig. 1.

\section{Reading CT data}

of wooden relics

\section{DL1 separating $\longrightarrow$ Color $\Rightarrow$ 3D reconstruction}

Fig. 1. 3D Visualization Flow Chart

\section{Experimental Analysis}

To verify the validation of the $3 \mathrm{D}$ visualization method of the wooden specimen, it was analyzed by MATALAB on Windows 7 for image segmentation. The N1 specimen was scanned vertically using the Philips Brilliance 16 CT (Model A), the layer thickness was set as $0.8 \mathrm{~mm}$, and 700 vertical-section CT images in Dicom format were obtained. 


\section{Section CT image segmentation and generation}

The vertical section CT images of the specimen were segmented using 3DL1. The $128^{\text {th }}, 148^{\text {th }}$, and $168^{\text {th }}$ vertical section CT images (Fig. 2) from the 700 images were selected for the segmentation to remove the non-specimen background, the resulting images are shown in Fig. 3.

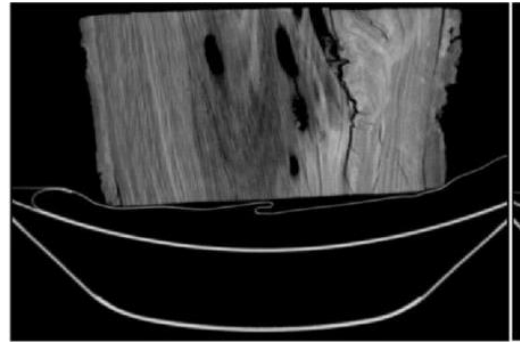

A $\left(128^{\text {th }}\right)$

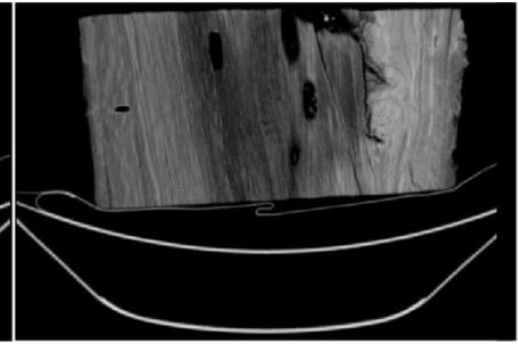

B $\left(148^{\text {th }}\right)$

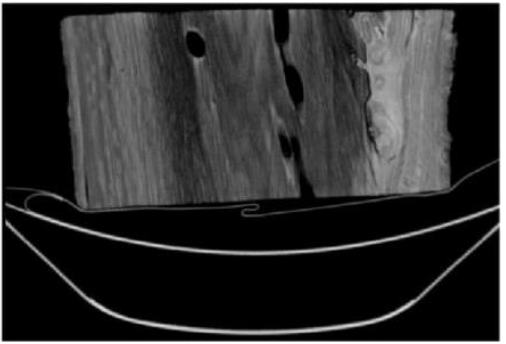

C $\left(168^{\text {th }}\right)$

Fig. 2. Original CT images of wood specimens (vertical section); A: $128^{\text {th }}$ cross-section; B: $148^{\text {th }}$ cross-section; C: $168^{\text {th }}$ cross-section

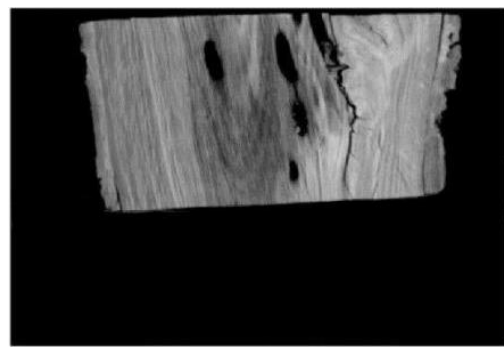

A $\left(128^{\text {th }}\right)$

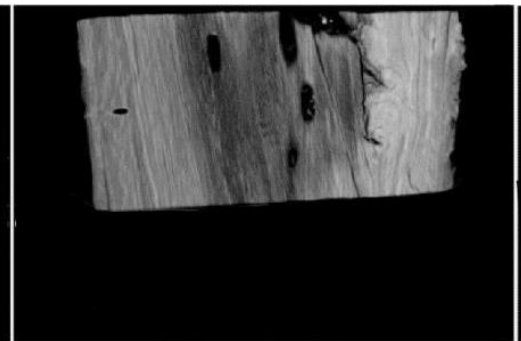

B $\left(148^{\text {th }}\right)$

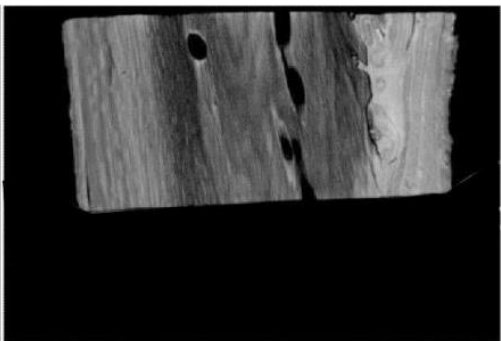

C $\left(168^{\text {th }}\right)$

Fig. 3. Partial CT images after 3DL1 segmentation (vertical section); A: $128^{\text {th }}$ cross-section; $B$ : $148^{\text {th }}$ cross-section; C: $168^{\text {th }}$ cross-section

The cross-section CT images of the specimen were generated from the vertical section images using the diagnostic image processing software. The Dicom format CT images were input into the software and 511 cross-section CT images were obtained. The 225th, 245th, and 265th cross-sections are shown in Fig. 4.

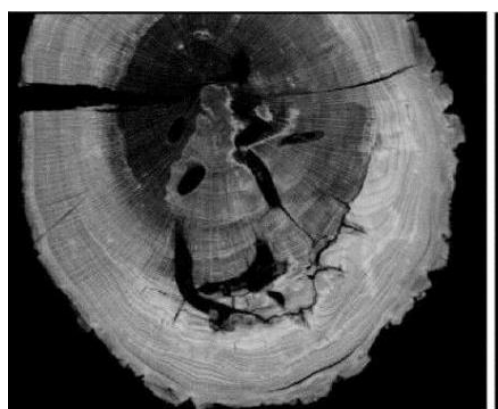

A (225th)

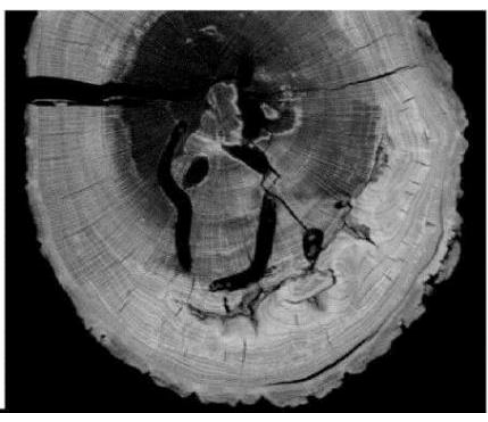

B (245th)

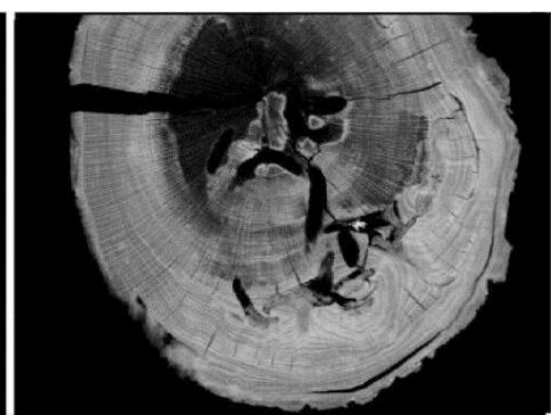

C (265th)

Fig. 4. Using diagnostic image processing software, cross-sectional CT images of specimens were generated from vertical section images; A: 225th cross-section; B: 245th cross-section; C: 265th cross-section 


\section{Coloring}

In order to obtain a 3D digital model of the defects in the specimen, the vertical and cross-section images were manually colored. The purpose of coloring process was to distinguish defects, such as wormholes and cracks, from the specimen original component. The defect boundary was manually identified according to the section CT image Dicom data, and then the defect area was colored. With different pixel information after coloring, defects and original components can be recognized during the 3D reconstruction process.

Cracks, wormholes, and nail holes are all hollow defects in the specimen and present similar grayscale threshold in the Dicom data; hence the same coloring method is applied on these defects. Random examples of colored wormholes in vertical and crosssections are shown in Figs. 5 and 6, respectively.

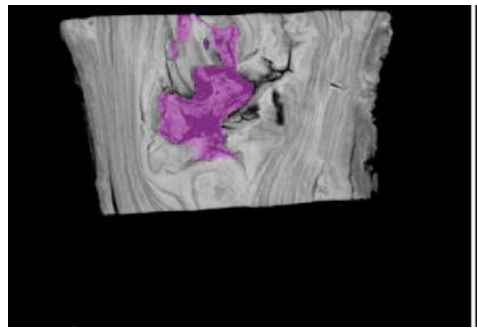

A $\quad\left(88^{\text {th }}\right)$

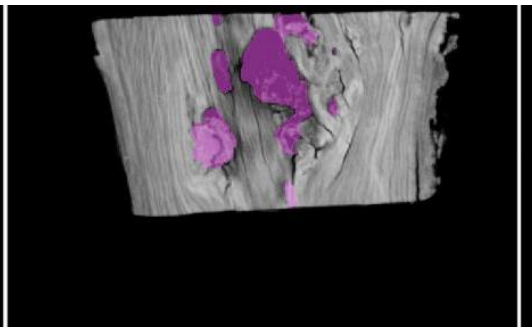

B $\left(108^{\text {th }}\right)$

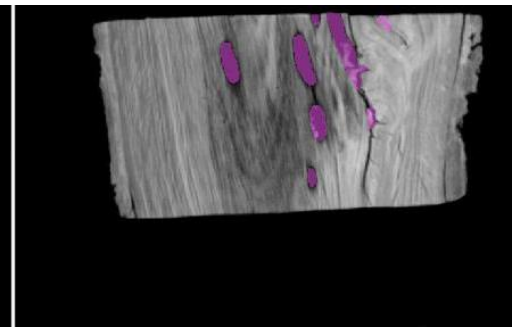

C $\left(128^{\text {th }}\right)$

Fig. 5. Wormhole coloring of CT images (vertical-section); A: $88^{\text {th }}$ cross-section; B: $108^{\text {th }}$ crosssection; C: $128^{\text {th }}$ cross-section

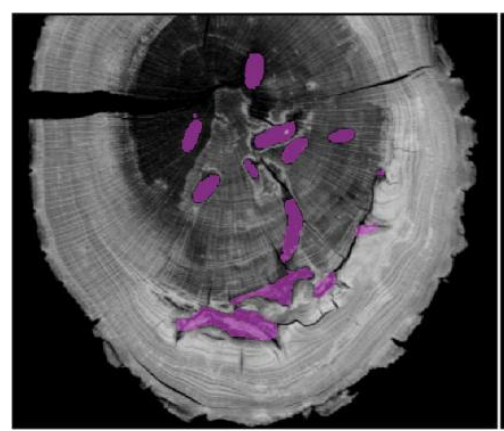

A (205th)

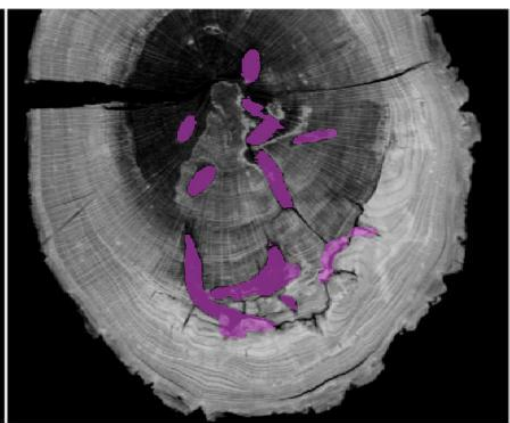

B (225th)

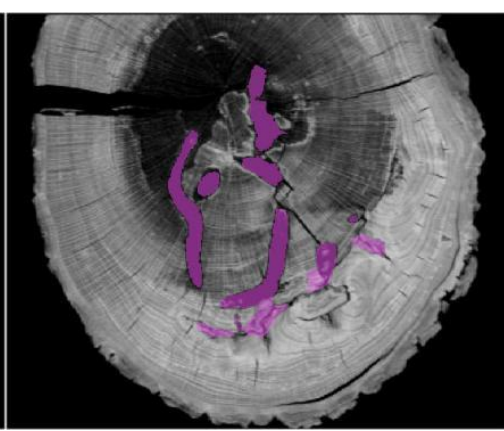

C (245th)

Fig. 6. Wormhole coloring of CT images (cross-section); A: 205th cross-section; B: 225th crosssection; C: 245th cross-section

The staining of wormholes and cracks in wood was more objective because the boundary between the cavity and the surrounding wood was clear. However, the hand coloring of rotten wood has certain subjectivity. Because the decay part of wood is processed by observing the color change of the image, the darker the color, the more serious the decay. But the boundary between the rotten part and the surrounding wood is not clear. As shown in Figs. 7 and 8, the decay from the center to the periphery is a gradual process from heavy to light. Therefore, the color will be subject to the subjective influence of the experimenter. At present, there is no standard to judge the degree of decay. 


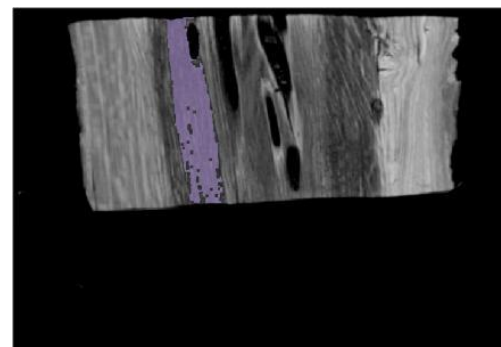

A $\left(188^{\text {th }}\right)$

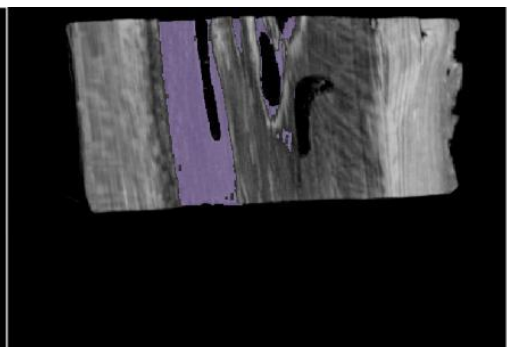

B $\quad\left(208^{\text {th }}\right)$

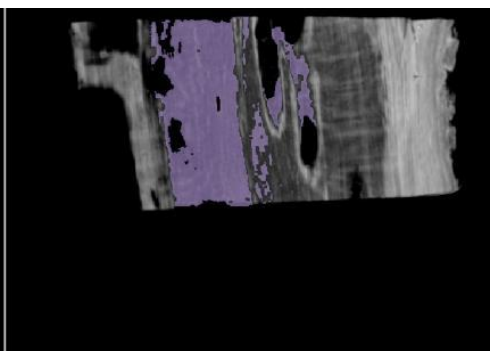

C $\left(228^{\text {th }}\right)$

Fig. 7. Colored decayed parts of the wooden specimen (vertical section); A: 188th cross-section; B: 208th cross-section; C: 228th cross-section

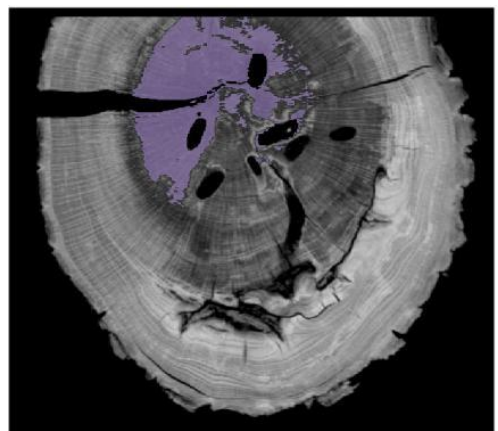

A (205th)

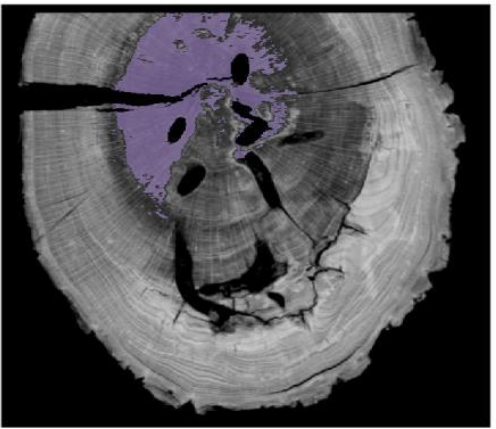

B (225th)

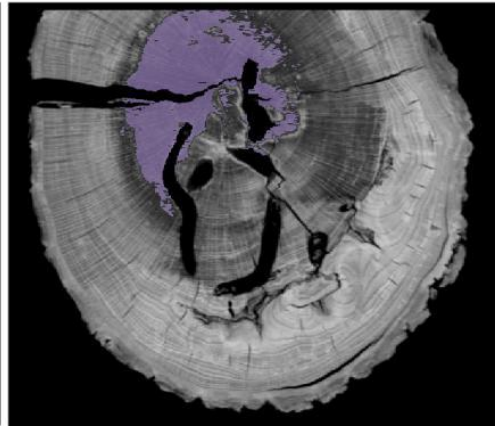

C (245th)

Fig. 8. Colored decayed parts of the wooden specimen (cross-section)

To color the borer excrement, the air in the hollow part of the wormhole needs to be colored, and then it is possible to subtract the borer excrement according to the Boolean operation to obtain a 3D model of the borer excrement.

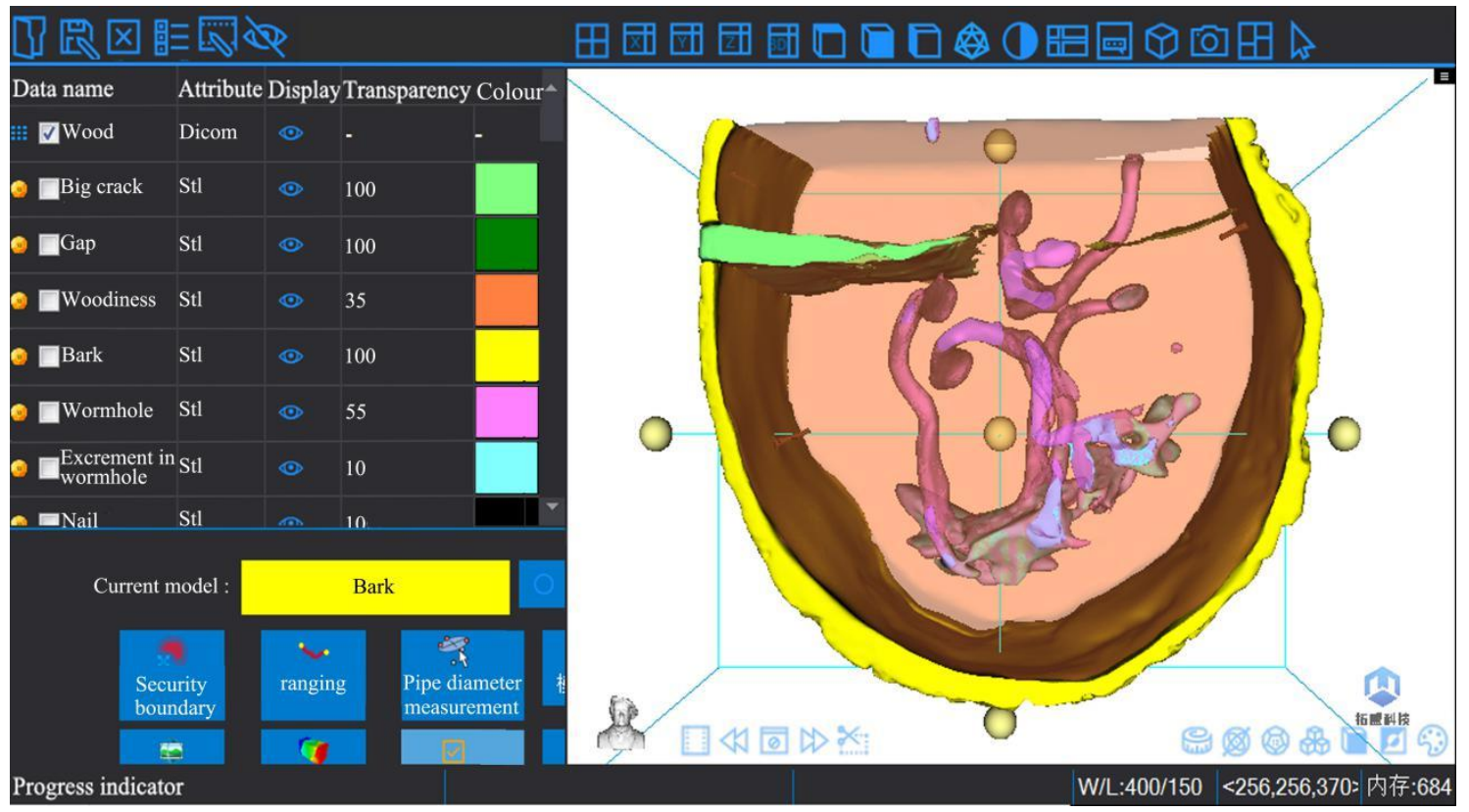

Fig. 9. 3D morphology of defects in wooden specimen 


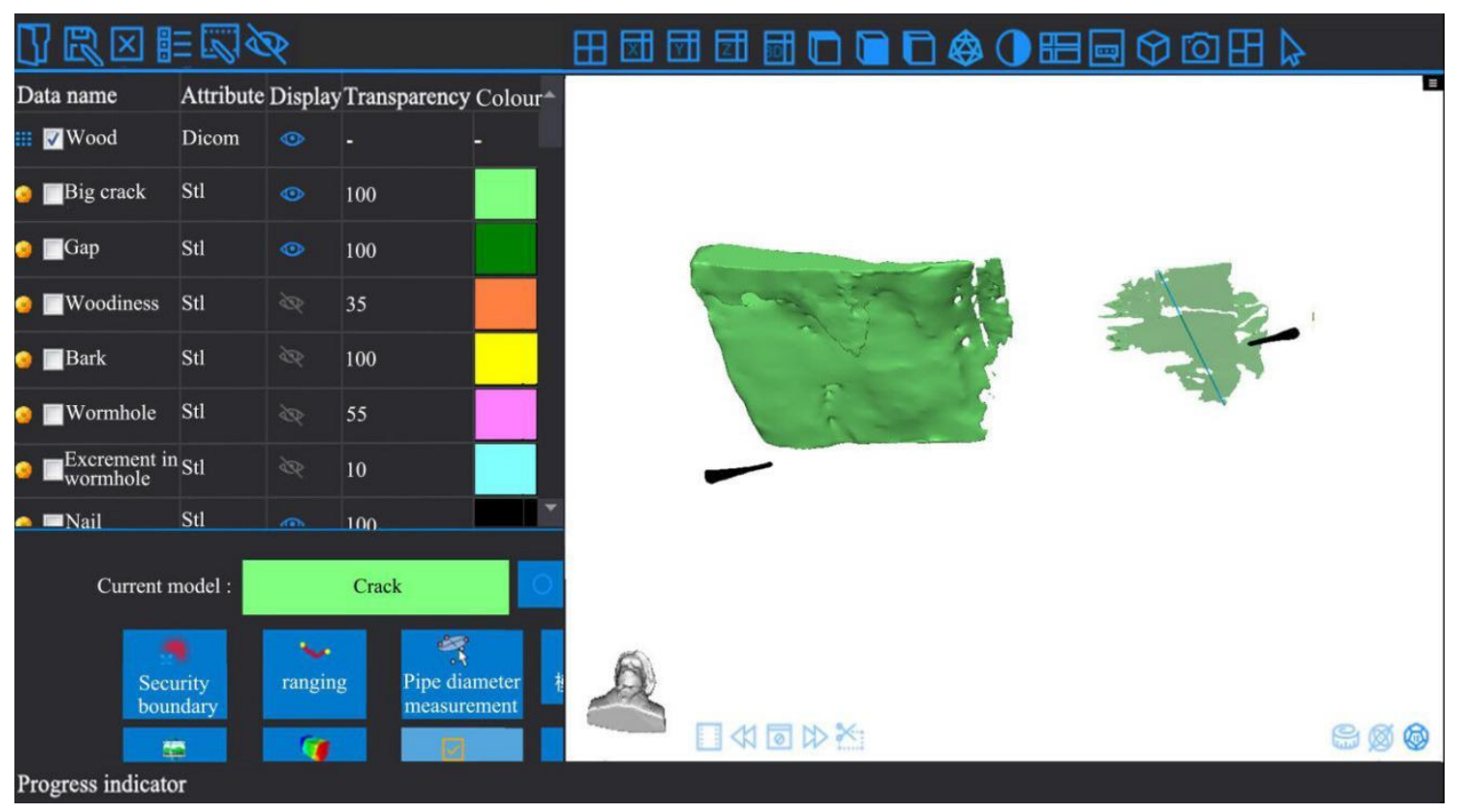

Fig. 10. 3D morphology of large cracks, small cracks, and nail holes in the wooden specimen

\section{$3 D$ reconstruction}

The 3D reconstruction is completed via the self-developed software to perform 3D modeling of the colored CT images. The transparency of the wooden part can be set to a lower value to facilitate the observation of the 3D models of each type of defects. As shown in Fig. 9, if the transparency was 35, the 3D morphology of defects, such as large cracks, small cracks, nail holes, wormholes, and borer excrement can be seen clearly.

\section{RESULTS AND DISCUSSION}

In order to further visually and clearly observe the 3D morphology of defects in specimen, each type of defect/original component can be displayed individually by hiding other parts in the self-developed software. For example, the 3D morphology of cracks and nail holes is shown in Fig. 10 when the bark, wormholes, and borer excrement are hidden. Another function of this 3D model is to display all defects in one image for labelling and size measurement (Figs. 11 and 12). However, most of the defects in wood are irregular, so it is impossible to calculate the defect volume with the traditional physical formula. Therefore, how to calculate the volume of the irregular defects through the pixel value is the focus of the next stage research.

With the same CT scanning equipment used in sample 1, the same scanning parameters were set to scan sample 2. $275 \mathrm{CT}$ images were obtained. Using the acquired CT image, the 3D shape of the crack in sample 2 was obtained by $3 \mathrm{D}$ reconstruction of the crack in sample 1. The 3D shape of the crack is shown in Fig. 12. 


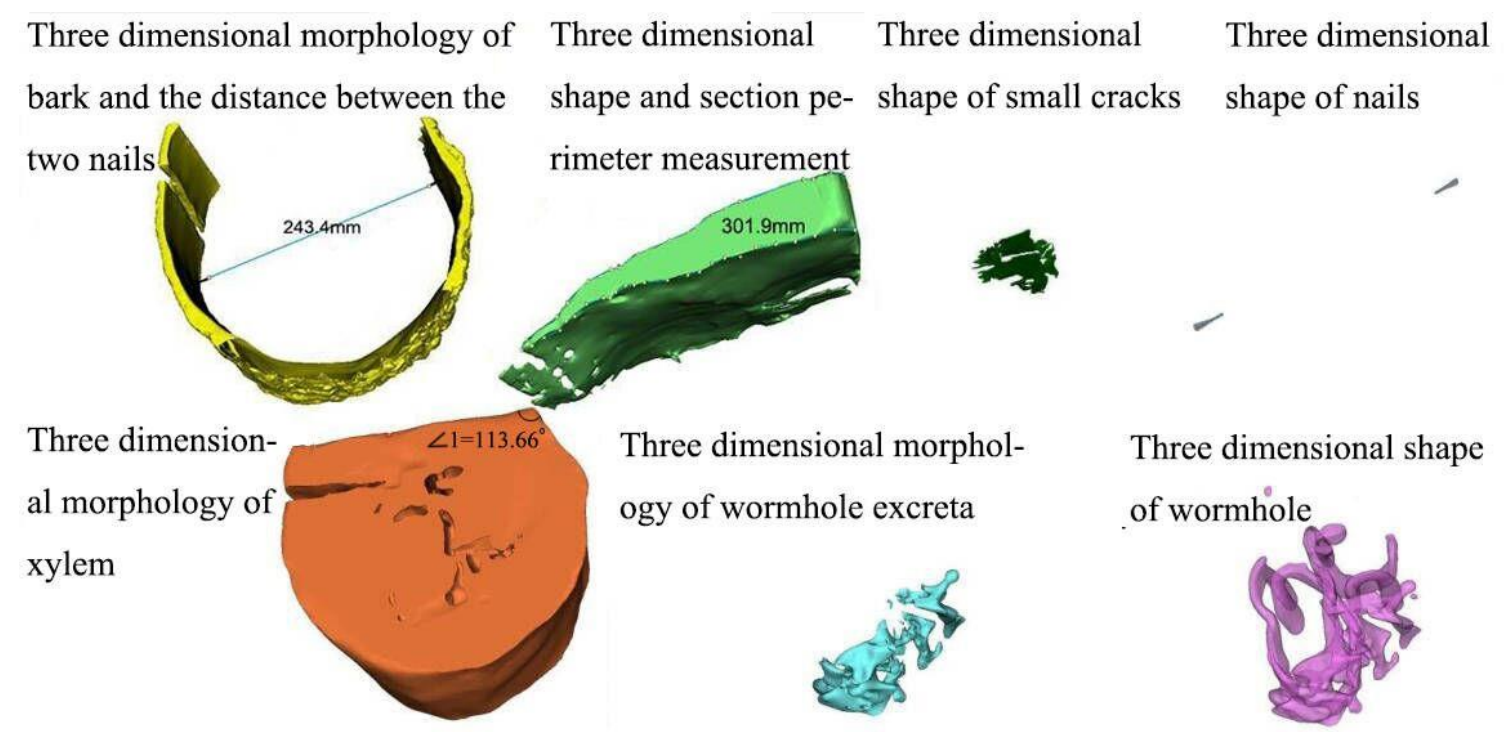

Fig. 11. 3D morphology and size information of each defect in the wooden specimen
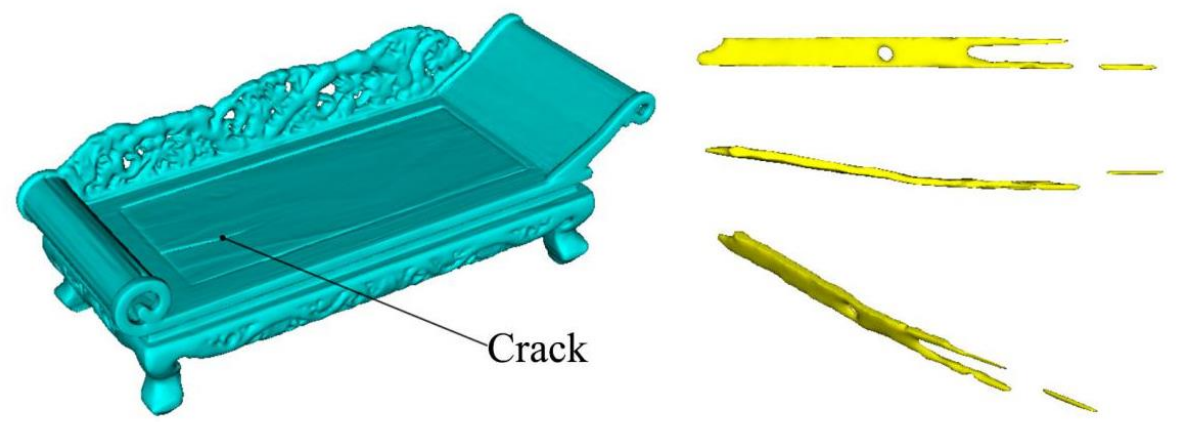

Fig. 12. 3D model of specimen $\mathrm{N} 2$ and multi-angle 3D diagram of crack part

The current coloring process requires the participation of professional personnel, and the CT machine has a large size and weight, which limits the application conditions of this method. Furthermore, the CT equipment used in this study is for medical tests, such that the capacity of the scanning chamber restricts the size of specimens to be tested. All these factors impede the application of this method.

\section{CONCLUSIONS}

1. The authors' method can visualize the complex morphology of defects inside wooden products and can accurately measure their size information at any position and any angle. Through accurate 3D morphology analysis of the location, shape, and size of defects, an intuitive, precise, customized, and scientific restoration protocol of wooden products and cultural relics could be provided.

2. The authors' method can generate accurate 3D morphology information of defects in wooden products, while the volume of each defect cannot be calculated yet due to the irregular shape and size of each defect. This method is limited to the size of the 
specimen, and the next step is to focus on the image acquisition method of the related specimen of mobile CT.

\section{ACKNOWLEDGMENTS}

This work was supported by "Heilongjiang philosophy and Social Science Foundation" (2020.9-2021.12) (grant number 20YSB056, China).

\section{REFERENCES CITED}

Brodersen, C. R. (2013). "Visualizing wood anatomy three dimensions with highresolution x-ray micro-tomography (MCT): A review," Iawa. J. 34(4), 408-424.DOI: $10.1163 / 22941932-00000033$

Chang, L. H., Chang, X. H., Chang, H., Qian, W., and Cheng, L. T. (2021). “The predictive research of ancient wooden building internal defects compressive bearing capacity based on nondestructive testing methods," Mech. Adv. Mater. Struc. 28(3), 252-259. DOI: 10.1080/15376494.2018.1556826

Chen, F. X., Feng, H. L., Du, X. C., Fang, Y. M., and Weng, X. (2015). “Threedimensional stress wave imaging method of wood internal defects based on TIDW," Chinese Journal of Sensors and Actuators, (in Chinese) 28(11), 1625-1633. DOI:10.3969/j.issn.1004-1699.2015.11.009

Chen, Y. P., and Guo, W. J. (2016). "Mechanical properties evaluation of two wood species of ancient timber structure with nondestructive testing methods," BioResources 11(3), 6600-6612.DOI:10.15376/biores.11.3.6600-6612

Du, X. C., Feng, H. L., Hu, M. Y., Fang, Y. M., and Chen, S. Y. (2018). “Threedimensional stress wave imaging of wood internal defects using TKriging method," Comput. Electron. Agr.148,63-71. DOI:10.1016/j.compag.2018.03.005

Ekevad, M. (2004). "Method to compute fiber directions in wood from computed tomography images," J. Wood. Sci. 50(1), 41-46. DOI:10.1007/s 10086-003-0524-Z

Gao, X., Li, Q. D., Cheng, W. L., Han, G. P., and Xuan, L. H. (2018). "Effects of moisture content, wood species, and form of raw materials on fiber morphology and mechanical properties of wood fiber-HDPE composites," Polym. Composite. 39(9), 3236-3246. DOI:10.1002/pc.24336

Hasegawa, M., Matsumura, J., Kusano, R., Tsushima, S., Sasaki, Y., and Oda, K. (2010). "Acoustoelastic effect in Melia azedarach for nondestructive stress measurement," Constr. Build. Mater, 24(9), 1713-DOI:1717.10.1016/j.conbuildmat.2010.02.018

Li, G. H., Weng, X., Du, X. C., Wang, X. P., and Feng, H. L. (2016). "Stress wave velocity patterns in the longitudinal-radial plane of trees for defect diagnosis," Comput. Electron. Agr. 124, 23-28. DOI: 10.1016/j.compag.2016.03.021

Marino, R. A., Fernandez, M. E., Fernandez-Rodriguez, C., and Mendez, M. (2010). "Detection of pith location in chestnut lumber (Castanea sativa Mill.) by means of acoustic tomography and longitudinal stress-wave velocity," Eur. J. Wood Wood Prod. 68(2), 197-206. DOI:10.1007/s00107-009-0366-5

Silva, F., Higuchi, N., Matos, J. M., Paula, E. M., and Santos, J. (2014). "Nondestructive evaluation of hardness in tropical wood," J. Trop. For. Sci. 26(1), 69-74. 
Tsarouchas, D., and Markaki, A. E. (2011). "Extraction of fibre network architecture by $\mathrm{X}$-ray tomography and prediction of elastic properties using an affine analytical model," Acta Materialia 59(18), 6989-7002. DOI: 10.1016/j.actamat.2011.07.051

Wei, X. W., Sun, L. P., Sun, Q. S., Xu, S. Z., Zhou, H. J., and Du, C. X. (2019). "Propagation velocity model of stress wave in longitudinal section of tree in different angular directions," BioResources 14(4), 8904-8922. DOI: 10.15376/biores. 14.4.8904-8922

Zhao, D. D., Liu, X. E., Yang, S. M., Yu, S., Tian, G. L., Ma, J. F., and Wang, Q. P. (2015). "Study on non-destructive testing of Guqin interior structure based on computed tomography," Spectrosc. Spect. Anal. 35, 3519-3523. DOI:10.3964/j.issn.1000-0593(2015)12-3519-05

Zhao, G. L., Deng, Z. J., Shen, J., Qiu, Z. W., and Huang, J. (2017). “Three-dimensional reconstruction of wood carving cultural relics based on CT tomography data," in: Third International Conference of Pioneering Computer Scientists, Engineers and Educators, ICPCSEE Proceedings, Springer Verlag, 462-471.

Zhao, G. L., Qiu, Z. W., Shen, J., Deng, Z. J., Gong J. H., and Liu, D. (2018). "Internal structural imaging of cultural wooden relics based on three-dimensional computed tomography," BioResources 13(1), 1548-1562. DOI:10.15376/biores.13.1.15481562

Article submitted: February 26, 2021; Peer review completed: June 26, 2021; Revised version received and accepted: July 9, 2021; Published: July 27, 2021.

DOI: 10.15376/biores.16.3.6267-6280 


\section{APPENDIX ALGORITHM INTRODUCTION}

\section{D TV-L1}

Brodersen (2013) proposed the following edge-weighted total variation norm:

$$
T V_{g}(u)=\int_{\Omega} g|\nabla u| d x d y
$$

where $g$ is an edge detection function, which can be chosen as $g=\exp \left(-\alpha|\nabla f|^{\beta}\right)$. The author suggests that $\alpha=10$ and $\beta=0.55$ suits well for different segmentation tasks. Theoretically, if $u$ is a characteristic function $1_{c}$ (i.e. $1_{c}$ is a binary image and $C$ denotes its boundary), this equation is equivalent to the geodesic active contour (GAC) segmentation model. However, the advantage of this model over GAC is that it can become a convex functional if variable $u$ is relaxed to interval $[0,1]$. This means one can find the global minimizer of Eq. 1 regardless of the initializations of $u$. The final segmentation can be then extracted from the optimized $u$ by choosing a level set within the range $[0,1]$.

The above edge-weighted TV norm (5) is used together with the L1 data fitting term for 2D image segmentation. When $u$ is the characteristic function $1_{c}$, the edgeweighted TV-L1 minimization problem can expressed as

$$
\min _{u}\left\{\lambda \int_{\Omega} g\left|\nabla 1_{C}\right| d x d y+\int_{\Omega}\left|1_{C}-f\right| d x d y\right\}
$$

where the variable $f \in[0,1]$ is an input 2D image and $l$ is a smoothing parameter, controlling the smoothness of the output image $u$. Note that minimization problem is not strictly convex, indicating that more than one global minimum may exist. In addition, model (2) is computationally expensive due to two L1 norms used in the formulation. Moreover, it cannot be used to segment volume data. The three issues can be addressed in the next section.

\section{D TV-L1 Segmentation Model and Fast Primal-Dual Projection}

We now extend (2) to the 3D case, which can be easily done by changing its integrated region from $x y$ domain to the $x y z$ domain.

$$
\min _{u \in[0,1]}\left\{\lambda \int_{\Omega} g|\nabla u| d x d y d z+\int_{\Omega}|u-f| d x d y d z\right\}
$$

Note that $u$ is no longer a characteristic function in (3) because it varies continuously between $[0,1]$. Next, we introduce an auxiliary variable $v$ to decouple (3) and reformulate it to become a strictly convex problem as follows

$$
\min _{u \in[0,1], v}\left\{\lambda \int_{\Omega} g|\nabla u| d \Omega+\int_{\Omega}|v| d \Omega+\frac{1}{2 \theta} \int_{\Omega}|u+v-f|^{2} d \Omega\right\}
$$

where $d \mathrm{~W}=d x d y d z$ and $q$ is a positive penalty parameter. Mathematically, if $\theta \rightarrow 0$, then model (4) is exactly the same as the TV-L1 model (3). However, practice has shown that the algorithm is robust even when $q$ is large. Note that the problem (4) has now become a convex optimization problem with respect to two variables $u$ and $v$, meaning that it allows to compute the global minimizers of them. More importantly, (4) can be solved efficiently 
with a fast algorithm. To do so, an alternating minimization technique is performed on the two variables sequentially.

1. We solve Equation (4) for $u$ by fixing $v$. The resulting energy functional to be minimized is

$$
\min _{u \in[0,1]}\left\{\lambda \int_{\Omega} g|\nabla u| d \Omega+\frac{1}{2 \theta} \int_{\Omega}|u+v-f|^{2} d \Omega\right\}
$$

This minimization problem is in fact exactly the ROF model [5, 6]. The only difference to the original ROF model lies in the edge-weighted TV norm. We can solve this problem using the fast primal-dual projection method proposed in [7] with an extrapolation step as follows

$$
\left\{\begin{array}{l}
\xi^{k+1}=\operatorname{Proj}_{K}\left(\xi^{k}-\sigma \lambda \nabla U^{k}\right) \\
u^{k+1}=f-v-\lambda \theta \nabla \cdot \xi^{k+1} \\
U^{k+1}=u^{k+1}+\alpha\left(u^{k+1}-u^{k}\right)
\end{array}\right.
$$

where $\xi$ is the dual variable and $\sigma$ is a time step which ensures that the scheme remains stable. In 3D $\sigma \leq 1 / 6$ dimension case to guarantee convergence of the algorithm. $\alpha \in(0,2)$ is an over or under-relaxation factor and in the paper, we set $a=1 . \operatorname{Pr} j_{K}$ to denote the back projection on set $K(i . e .\{x, y, z|| \xi(x, y, z) \mid \leq 1\})$ and it is defined as $\operatorname{Proj}_{K}(\xi)=\frac{\xi}{\max (|\xi|, g)}$

2. We solve Equation (4) for $v$ by fixing $u$. The resulting energy functional to be minimized is

$$
\min _{v}\left\{\int_{\Omega}|v| d \Omega+\frac{1}{2 \theta}|u+v-f|^{2} d \Omega\right\}
$$

which can be easily solved by the one-dimensional analytical shrinkage scheme below.

$$
v=\left\{\begin{array}{cl}
f-u-\theta & \text { if } f-u \geq \theta \\
f-u+\theta & \text { if } f-u \leq-\theta \\
0 & \text { if }|f-u| \leq \theta
\end{array}\right.
$$

\section{Discretization}

In order to implement the fast primal-dual algorithm for the TV-L1 model, the 3D discrete first order gradient and divergence differential operators are required. In this paper, a forward-back word finite difference scheme was used for these two operators. Specifically, let $\Omega \rightarrow R^{M N L}$ denotes the $3 \mathrm{D}$ grid space of the size $M N L$. The forward discrete gradient of a volume $u$ at a voxel $i, j, k$ reads

$\nabla u_{i, j, k}^{+}=\left(\partial_{x} u_{i, j, k}^{+}, \partial_{y} u_{i, j, k}^{+}, \partial_{z} u_{i, j, k}^{+}\right)$

where,

$\partial_{x} u_{i, j, k}^{+}$

$\partial_{x} u_{i, j, k}^{+}$

$\partial_{x} u_{i, j, k}^{+}$ 
The 3D divergence is computed with a backward scheme: for the dual vector $\xi=\left(\xi^{1}, \xi^{2}, \xi^{3}\right)$, we have

$$
(\operatorname{div} \xi)_{i, j, k}=\left\{\begin{array}{ll}
p_{i, j}^{1} & j=1 \\
p_{i, j}^{1}-p_{i, j-1}^{1} & 1<j<N \\
-p_{i, j-1}^{1} & j=N
\end{array}+\left\{\begin{array}{ll}
p_{i, j}^{2} & i=1 \\
p_{i, j}^{2}-p_{i-1, j}^{2} & 1<i<M \\
-p_{i-1, j}^{2} & i=M
\end{array}+ \begin{cases}p_{i, j}^{2} & i=1 \\
p_{i, j}^{2}-p_{i-1, j}^{2} & 1<i<M \\
-p_{i-1, j}^{2} & i=M\end{cases}\right.\right.
$$

Having defined all necessary discrete quantities, the numerical optimization for two sub-problems (5) and (8) can be implemented practically. For clarity, we present the flow chart of the primal-dual projection for the 3D TV-L1 model is shown in Table1. Note that there is no need to exactly solve this sub-optimization problem (1). One iteration $(k=1)$ of this scheme is sufficient to make the entire algorithm converge. Note that as the input $f$ has been stretched into [0,1], the constraint $u \in[0,1]$ can be omitted. To obtain the final segmentation, a level set of $u$ is selected as $u=0.5$

Table 1. Primal-Dual Projection Algorithm for TV-L1 Model

\begin{tabular}{|ll|}
\hline Steps & \multicolumn{1}{|c|}{ Method } \\
\hline 1. & Initialization: linear stretch ${ }^{f}$ to $[0,1], v^{0}=f, \xi^{0}=0$ and $u^{0}=0$ \\
\hline 2 & Repeat \\
\hline 3 & Compute dual variable ${ }^{\xi}$ using (10) \\
\hline 4 & Compute primal variable ${ }^{u}$ using (10); \\
\hline 5 & Compute extrapolating variable $U$ using (10); \\
\hline 6 & Compute ${ }^{v}$ using (12); \\
\hline 7 & Until the stopping criterion is satisfied. \\
\hline
\end{tabular}

